\title{
Yeast Protein
}

National Cancer Institute

\section{Source}

National Cancer Institute. Yeast Protein. NCI Thesaurus. Code C18474.

Proteins found in any species of yeast. 